\title{
The Effects of Different Types of Strength Training for Recreational Purposes on the Body Composition and Strength Development of University Students
}

\author{
Serhat Özbay \\ Faculty of Sport Science, Erzurum Technical University, Erzurum, Turkey. \\ Email:serhat.ozbay@erzurum.edu.tr
}

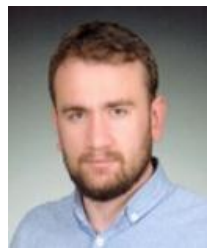

\begin{abstract}
The aim of this study was to investigate the effect of CrossFit and resistance training on maximal strength, isometric strength and strength continuity in recreational student athletes. 28 healthy male volunteer students who were doing sport for recreational purposes participated in the study. After the participants were randomly divided into two groups, strength tests were performed and body composition measurements were taken before and after the 16-week training period. Bench press and squat were applied to determine maximal force; leg and handgrip strength were applied to determine isometric force; pull-up and push-up tests were applied to determine the continuity of strength. Body composition measurements were performed with electronic scales. Statistical analyses were performed with SPSS 21.0 program. Significance level was accepted as $p<0.05$. The CrossFit training program included consecutive 5 pull-ups, 10 push-ups and 15 squat exercises for 20 minutes (CrossFit-Cindy). Resistance training program consisted of 3 sets of bench press, lat pull down, leg press, biceps curl and triceps extension exercises with 3 minute intervals using $70 \%$ of the weights the participants could perform with pre-determined maximum 1 repetition. The results of the study showed that body fat percentages of both groups decreased significantly, performance variables increased significantly and body weight did not change significantly. In addition, the increase in push-up exercise was higher in the CrossFit group than in the resistance group. Thus, this study shows that although CrossFit training is short-termed, it can be used as an alternative to classical resistance training.
\end{abstract}

Keywords: Cross Fit, Resistance exercises, Strength, Body composition, Recreational, Training.

Citation | Serhat Özbay (2019). The Effects of Different Types of Strength Training for Recreational Purposes on the Body Composition and Strength Development of University Students. Asian Journal of Education and Training, 5(2): 381-385. History:

Received: 26 March 2019

Revised: 1 May 2019

Accepted: 7 June 2019

Published: 9 August 2019

Licensed: This work is licensed under a Creative Commons

Attribution 3.0 License (cc)

Publisher: Asian Online Journal Publishing Group
Funding: This study received no specific financial support.

Competing Interests: The author declares that there are no conflicts of interests regarding the publication of this paper.

Transparency: The author confirms that the manuscript is an honest, accurate, and transparent account of the study was reported; that no vital features of the study have been omitted; and that any discrepancies from the study as planned have been explained.

Ethical: This study follows all ethical practices during writing.

\section{Contents}

1. Introduction

References. 


\section{Contribution of this paper to the literature}

This study contributes to the existing literature by investigating the effect of CrossFit and resistance training on maximal strength, isometric strength and strength continuity in recreational student athletes.

\section{Introduction}

Recreational sports are activities which are done to meet the individual's needs (entertainment, health, aesthetics, psychological) without expecting any performance output (professional success). The content of recreational sports may vary according to the individual's expectations. Some individuals take part in activities that include recreational hiking, water sports, extreme sports, team or individual sports, while others prefer the fitness center. In all of these regular activities, the individual gets positive physiological and psychological benefits. Regular activities, especially those done in fitness centers, result in an increase in strength output.

Strength is an important component in many sports (Ribeiro et al., 2017; Colquhoun et al., 2018; Rajkumar and Divya, 2018). Resistance exercises, which are mostly preferred for strength development, are frequently used in sports sciences, especially in research designs where the effects of any intervention, such as training, are examined (Weir, 2005; Ritti-Dias et al., 2011; Özbay and Ulupınar, 2018).

CrossFit $^{\circledR}$ (CrossFit, Inc., Washington, DC, USA) is a high intensity functional training model that has become very popular in recent years (Butcher et al., 2015). CrossFit is one of the fastest growing functional methods of high intensity training. It is thought that more than 200,000 athletes participate in CrossFit training in approximately 11,000 gyms (Maté-Muñoz et al., 2018).

When the literature was examined, significant benefits of CrossFit trainings were found on health and wellness (Glassman, 2006; Sparkes and Behm, 2010; Kliszczewicz et al., 2014; Fernández et al., 2015; Eather et al., 2016; Sousa et al., 2016; Yüksel et al., 2019). CrossFit training also affects both aerobic and anaerobic performance (Smith et al., 2013). Basically, CrossFit is a training model in which high-intensity functional exercises are combined with cardiovascular exercises and gymnastics, body weight and weights (Glassman, 2010; Sibley, 2012; Gregory et al., 2017). Programs are usually in the form of "workout of the day" (WOD). WOD consists of different, functional motion models. They can be scaled regardless of the participants' strength and form level (Eather et al., 2016; Dilber and Doğru, 2018). High intensity interval training and body weight workout ranks among the top three in 2017 worldwide sports trend list Thompson (2017).

There are many factors affecting strength and development. One of these factors is the proportion of body fat and body fat percentage. Strength training not only increases muscle resistance, but also regulates the composition of the body. Two muscles with the same circumference and volume produce different amounts of strength due to the different fatty tissue they contain. Fat impedance not only reduces the contraction force but also limits the size and speed of contraction of the muscle fibres by creating friction (viscosity). As a result, excess fat in the body means extra burden as it will cause extra energy consumption to transport fat for performance (Tamer, 1991; Yüksel et al., 2017).

The aim of this study is to examine the effects of CrossFit and resistance training on maximal strength, isometric strength, strength continuity and body composition in students who do sport for recreational purposes and to show whether these training methods are alternative for each other.

\section{Method}

\subsection{Participants}

28 healthy male volunteer students doing sport for recreational purposes participated in the study. After the participants were divided into groups, the differences between anthropometric measurements and 1TM loads were tested and if the difference was significant, the groups were randomly redistributed. The participants were randomly divided into two groups [CrossFit Group $(n=14)$, Resistance Group $(n=14)$ ], strength and anthropometric measurements were performed before and after the 16-week training period. The participants did not receive any additional food or nutritional supplements during the training period. They were selected on a voluntary basis from individuals without temporary disability or disease. They were provided with detailed explanations and they signed informed consent prior to volunteering for the study. All this process was conducted according to the Helsinki Declaration.

\subsection{Anthropometric Measurements}

The height of the participants were measured by stadiometer (Holtain Ltd., UK); weight, body mass index $(\mathrm{BMI})$ and body fat percentage $(\mathrm{BF} \%)$ were measured by bioelectrical impedance analyser (Tanita TBF 401, Japan).

\subsection{Strength Tests}

Maximal dynamic strength of participants was measured using one repetition squat and bench press test. The wrestlers performed 3 trials for both test at 5 -min intervals. The load was increased by $3-10 \%$ in valid trials or reduced by $3-10 \%$ in invalid trials according to the request of the participants. For a trial to be considered valid, it was based on completing a full range of motion and returning to the starting position.

Isometric strengths of participants were measured via relevant dynamometers (Takei A5001 Hand Grip Dynamometer and Takei A5002 Leg Dynamometer, Tokyo, Japan). Dominant hand was preferred during the hand grip strength test. During the leg strength test, the pull-bar of the dynamometer was placed in the hands and the angle of the knees was set at 45 degrees. In both tests, the highest score from two trials with 3 -min rest intervals was considered valid.

Strength endurance performances of participants were determined via pull-up and push-up tests. For the pullup test to be valid, the total number of repetitions the participant was able to pull the chin above the constant bar were calculated. For the push-up test to be valid, the total number of repetitions the participant was able to complete full range of motion and to return to the starting position were calculated. 
1TM loads of the participants were determined on two different days after a practice workout. Rest periods of 2-3 days were used between these exercises. On the first day, 1TM loads of bench press and squat exercises were determined. On the second day, the same tests were repeated to ensure test-retest reliability. The higher load between the two test days was determined as the valid 1TM load. For each exercise, participants were given 5 rights with 5 minute intervals. The other exercise was started after a rest of at least 10 minutes. The same procedure was reapplied after 16 weeks of training to determine performance improvement.

\subsection{Workout Protocols}

CrossFit workout program was applied to the participants as Cindy model three days a week for 16 weeks. The workouts were made for 20 minutes without rest as consecutive 5 pull-ups, 10 push-ups and 15 squat exercises with body weights (Butcher et al., 2015). All workouts were observed by the researcher.

Resistance training, Resistance training group received resistance training for 16 weeks. Workouts were done three times a week. In resistance training, bench press, lat pull down, leg press, biceps curl and triceps extension exercises were applied in 3 sets with 3 minute intervals. The subjects attempted to achieve maximum repetition in $70 \%$ of their 1TM load while performing the exercises. Before starting the exercises, the subjects performed a warm-up of 15 repetitions in $50 \%$ of their load. All workouts were observed by the researcher.

\subsection{Statistical Analysis}

Statistical analyses were performed with SPSS 21.0 program. Significance level was accepted as $\mathrm{p}<0.05$. Mean and standard deviation were used for data analysis. Independent t-test was used to determine whether there was a difference between the groups at the beginning of the study. Paired t-test was used to determine the difference between the measured values of a group before and after the research period. In addition, two-way ANOVA test was used for repeated measurements to determine the group X time (before-after) interaction.

\section{Findings}

\begin{tabular}{|c|c|c|c|c|}
\hline $\begin{array}{c}\text { Descriptive and } \\
\text { performance }\end{array}$ & CrossFit Group $(n=14)$ & Resistance Group $(n=14)$ & $\mathbf{t}$ & $\mathbf{p}$ \\
\hline Age (year) & $20.8 \pm 2.0$ & $19.7 \pm 1.8$ & 1.475 & 0.152 \\
\hline Height $(\mathrm{cm})$ & $171.4 \pm 10.2$ & $170.1 \pm 9.2$ & 0.350 & 0.729 \\
\hline Weight (kg) & $66.5 \pm 9.3$ & $66.2 \pm 9.9$ & 0.074 & 0.941 \\
\hline BMI $\left(\mathrm{kg} / \mathrm{m}^{2}\right)$ & $23.2 \pm 1.9$ & $23.1 \pm 2.6$ & 0.074 & 0.941 \\
\hline Fat $\%$ & $9.1 \pm 2.3$ & $10.5 \pm 3.25$ & 1.383 & 0.178 \\
\hline $\mathrm{BP}(\mathrm{kg})$ & $70.1 \pm 10.1$ & $69.7 \pm 11.3$ & 0.088 & 0.930 \\
\hline SQ (kg) & $106.3 \pm 14.9$ & $105.9 \pm 15.9$ & 0.075 & 0.941 \\
\hline $\mathrm{LS}(\mathrm{kg})$ & $143.3 \pm 22.9$ & $136.6 \pm 23.0$ & 0.765 & 0.451 \\
\hline HGS (kg) & $38.0 \pm 3.9$ & $41.0 \pm 6.2$ & 1.614 & 0.119 \\
\hline push-up (repetition) & $15.6 \pm 3.1$ & $14.6 \pm 2.2$ & 1.318 & 0.199 \\
\hline pull-up (repetition) & $31.6 \pm 6.9$ & $34 . .8 \pm 5.6$ & 1.041 & 0.307 \\
\hline
\end{tabular}

Values were presented mean $\pm \mathrm{SD}$

$\mathrm{BP}=$ bench press; $\mathrm{SQ}=$ skuat; $\mathrm{LS}=$ leg strength; HGS = handgrip strength

Initial descriptive and performance characteristics of the groups were examined and no significant difference was found between the groups Table 1.

Table-2. Performance values of the participants before and after 16 weeks training period ( $\mathrm{N}=28$.

\begin{tabular}{c|c|c|c|c}
\hline \multicolumn{3}{c}{ CrossFit Group (n=14) } & \multicolumn{2}{c}{ Resistance Group (n=14) } \\
\hline & Before & After & Before & After \\
\hline Weight (kg) & $66.5 \pm 9.3$ & $66.3 \pm 9.5$ & $66.2 \pm 9.9$ & $66.4 \pm 10.6$ \\
\hline Fat\% & $9.4 \pm 2.3$ & $8.6 \pm 1.8^{*}$ & $10.8 \pm 3.2$ & $10.3 \pm 3.0^{*}$ \\
\hline BP (kg) & $70.1 \pm 10.1$ & $77.9 \pm 9.6^{*}$ & $69.7 \pm 11.5$ & $76.4 \pm 11.6^{*}$ \\
\hline SQ (kg) & $106.4 \pm 14.9$ & $114.9 \pm 14.5^{*}$ & $105.9 \pm 15.9$ & $114.2 \pm 17.1^{*}$ \\
\hline LS (kg) & $143.2 \pm 22.9$ & $152.7 \pm 20.9^{*}$ & $136.6 \pm 22.9$ & $145.1 \pm 23.7^{*}$ \\
\hline HGS (kg) & $38.0 \pm 3.9$ & $40.8 \pm 4.6^{*}$ & $41.1 \pm 6.1$ & $42.7 \pm 6.2^{*}$ \\
\hline push-up (repetition) & $15.6 \pm 3.1$ & $18.1 \pm 4.3^{*}$ & $14.6 \pm 2.2$ & $18.5 \pm 3.4^{*}$ \\
\hline pull-up (repetition) & $34.7 \pm 5.6$ & $44.1 \pm 6.6^{* \#}$ & $31.6 \pm 6.9$ & $38.4 \pm 7.9^{*}$ \\
\hline Vart
\end{tabular}

Values are presented mean \pm SD.

* There is significant difference between before and after values.

\# Group X time (before-after) interaction is significant.

$\mathrm{BP}=$ bench press; $\mathrm{SQ}=$ skuat; $\mathrm{LS}=$ leg strength; HGS = handgrip strength

Anthropometric measurements and performance values of the participants were monitored before and after the 16-week training period Table 2. While both groups showed a significant increase in all performance variables; body fat percentages decreased significantly; however, there was no significant change in body weight. In addition, the increase in push-up variable in the CrossFit group was significantly higher than the increase in the resistance group.

\section{Discussion and Conclusion}

The number of students doing sports for recreational purposes is increasing day by day. "CrossFit", which includes various types of workout, is a serious alternative for students doing recreational sports. This study was conducted to investigate the effect of CrossFit and resistance training on maximal strength, isometric strength and strength continuity in recreational student athletes and to show whether these training methods can be an 
alternative for each other. Anthropometric measurements and performance values of the participants were monitored before and after the 16-week training period Table 2. While there was a significant increase in all performance variables in both groups; a significant reduction was found in body fat percentages. However, there was no significant change in the body weight of the participants. In addition, it was found that the increase in pushup variable in the CrossFit group was significantly higher than the increase in the resistance group.

When studies about resistance training were examined, both CrossFit and classic resistance training methods were shown to have positive effects on body composition and strength increase. In their study, MurawskaCialowicz et al. (2015) stated that three month long CrossFit workout preformed with 15 young female participants decreased body fat percentage significantly (Murawska-Cialowicz et al., 2015). Fatoba (2016) found that the decrease in body fat percentage was significant at the end of 12-week-long circular resistance training program applied to elite volleyball players (Fatoba, 2016). In a study in which 6-week-long different strength training methods were applied on 33 female participants, Yüksel et al. (2017) reported that the decrease in body composition in both CrossFit and definition training program was significant (Yüksel et al., 2017). In a study conducted on young men and women, (Uçan, 2013) found significant change in body fat ratio as a result of 10-week resistance training, while no significant change was found in body weight and BMI (Body Mass Index) (Uçan, 2013). In their study they applied CrossFit-based high intensity strength program, Smith et al. (2013) found 3,7\% decrease in body fat percentage (Smith et al., 2013). According to the results of our study, it is thought that the greater decrease in fat ratio is due to the intensity of workout protocols and the differences in diets applied in the study. In their study they compared CrossFit training method and classical resistance training, Gerhart and Pasternostro (2014) found that the difference between was significant in favour of CrossFit (Gerhart and Pasternostro, 2014). On the other hand, in their study which included 60 participants, Barfield et al. (2012) did not find a significant difference in body mass index and hand grip strength (Barfield et al., 2012). In their study they applied CrossFit workout method, Dilber and Doğru (2018) reported a significant increase in body fat percentage, left and right grip and leg strength of the participants (Dilber and Doğru, 2018). In their study they applied CrossFit (Cindy) workout program on 32 participants for 8 weeks, Yüksel et al. (2019) found that this method developed strength ability (Yüksel et al., 2019).

In conclusion, it can be seen that both training methods have positive effects on body composition and strength. When it is considered that CrossFit training method, which has become very popular recently, can be designed for different purposes and is practical and saves time, it is thought that it is a serious alternative to resistance training in today's world.

\section{References}

Barfield, J., B. Channell, C. Pugh, M. Tuck and D. Pendel, 2012. Format of basic instruction program resistance training classes: Effect on fitness change in college students. Physical Educator, 69(4): 325.

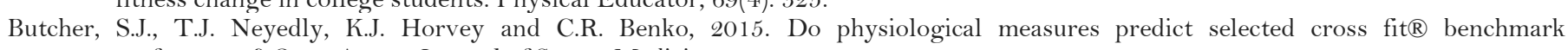
performance? Open Access Journal of Sports Medicine, 6: $241-247$.

Colquhoun, R.J., C.M. Gai, D. Aguilar, D. Bove, J. Dolan, A. Vargas, K. Couvillion, N.D. Jenkins and B.I. Campbell, 2018. Training volume, not frequency, indicative of maximal strength adaptations to resistance training. The Journal of Strength \& Conditioning Research, 32(5): 1207-1213.Available at: https://doi.org/10.1519/jsc.0000000000002414.

Dilber, A.O. and Y. Doğru, 2018. The effect of high-intensity functional exercises on anthropometric and physiological characteristics in sedantery. International Journal of Sport Exercise and Training Science, 4(2): 64-69.Available at: https://doi.org/10.18826/useeabd.425483.

Eather, N., P.J. Morgan and D.R. Lubans, 2016. Improving health-related fitness in adolescents: The CrossFit Teens ${ }^{\mathrm{TM}}$ randomised controlled trial. Journal of Sports Sciences, 34(3): 209-223.

Fatoba, M., 2016. Impact of resistance circuit training programme on the skinfolds of state elite volleyball players. International Journal of Science and Science Education, 6(1): 8-11.

Fernández, J.F., R.S. Solana, D. Moya, J.M.S. Marin and M.M. Ramón, 2015. Acute physiological responses during crossfit ${ }^{\circledR}$ workouts. European Journal of Human Movement, 35: 114-124.

Gerhart, D.H. and B.M. Pasternostro, 2014. A comparison of crossfit training to traditional anaerobic resistance training in terms of selected fitness domains representative of overall athletic performance. In International Journal of Exercise Science: Conference Proceedings, 9(2): 26.

Glassman, G., 2006. Validity of crossfit tested. The Cross Fit Journal Articles, 41: 1-4

Glassman, G., 2010. Cross fit training guide level 1, April; $2-3 . \quad$ Available from http://library.crossfit.com/free/pdf/CFJ_English_Level1_TrainingGuide.pdf [Accessed 15 June 2019].

Gregory, R.M., H. Hamdan, D. Torisky and J. Akers, 2017. A low-carbohydrate ketogenic diet combined with 6-weeks of crossfit training improves body composition and performance. International Journal of Sports and Exercise Medicine, 3(2): 1-10.Available at: https://doi.org/10.23937/2469-5718/1510054.

Kliszczewicz, B., R. Snarr and M. Esco, 2014. Metabolic and cardiovascular response to the cross fit workout 'cindy': A pilot study. Journal of Sport and Human Performance, 2(2): 1-9.

Maté-Muñoz, J.L., J.H. Lougedo, M. Barba, A.M. Cañuelo-Márquez, J. Guodemar-Pérez, P. García-Fernández, M.d.C. Lozano-Estevan, R. Alonso-Melero, M.A. Sánchez-Calabuig and M. Ruíz-López, 2018. Cardiometabolic and muscular fatigue responses to different crossfit ${ }^{\circ}$ workouts. Journal of Sports Science \& Medicine, 17(4): 668.

Murawska-Cialowicz, E., J. Wojna and J. Zuwala-Jagiello, 2015. Crossfit training changes brain-derived neurotrophic factor and irisin levels at rest, after wingate and progressive tests, and improves aerobic capacity and body composition of young physically active men and women. Journal Physiol Pharmacol, 66(6): 811-821.

Özbay, S. and S. Ulupınar, 2018. Reliability of $1 \mathrm{tm}, 5 \mathrm{tm}$ and $10 \mathrm{tm}$ tests in upper body resistance exercises. Turkish Journal of Sport Sciences, 2(1): $1-7$

Rajkumar, R. and K. Divya, 2018. Impact of specific training on selected speed, explosive power and muscular strength parameters among school men handball players. Indian Journal of Applied Research, 7(9): 587-588.

Ribeiro, A.S., B.J. Schoenfeld, S.J. Fleck, F.L. Pina, M.A. Nascimento and E.S. Cyrino, 2017. Effects of traditional and pyramidal resistance training systems on muscular strength, muscle mass, and hormonal responses in older women: A randomized crossover trial. The Journal of Strength \& Conditioning Research, 31(7): 1888-1896.

Ritti-Dias, R.M., A. Avelar, E.P. Salvador and E.S. Cyrino, 2011. Influence of previous experience on resistance training on reliability of onerepetition maximum test. The Journal of Strength \& Conditioning Research, 25(5): 1418-1422.

Sibley, B.A., 2012. Using sport education to implement a cross fit unit. Journal of Physical Education, Recreation \& Dance, 83(8): 4248.Available at: https://doi.org/10.1080/07303084.2012.10598829.

Smith, M.M., A.J. Sommer, B.E. Starkoff and S.T. Devor, 2013. Crossfit-based high-intensity power training improves maximal aerobic fitness and body composition. Journal Strength Cond Reserch, 27(1 1): 3159-3172.

Sousa, A.F.D., G.B.D. Santos, T.D. Reis, A.J. Valerino, S. Del Rosso and D.A. Boullosa, 2016. Differences in physical fitness between recreational crossfit巴 and resistance trained individuals. Journal of Exercise Physiology Online, 19(5): 112-122. 
Sparkes, R. and D.G. Behm, 2010. Training adaptations associated with an 8-week instability resistance training program with recreationally active individuals. The Journal of Strength \& Conditioning Research, 24(7): 1931-1941.Available at: https://doi.org/10.1519/jsc.0bo13e3181df7fe4.

Tamer, K., 1991. Measurement and evaluation of physical performance. Ankara: Gökçe Ofset Printing.

Thompson, W.R., 2017. Worldwide survey of fitness trends for 2018: The CREP edition. ACSM's Health \& Fitness Journal, 21(6): 10-19.

Uçan, Y., 2013. Effects of different types of exercises on body composition in young men and women. Life Science Journal, 10 (3): 1799-1806.

Weir, J.P., 2005. Quantifying test-retest reliability using the intraclass correlation coefficient and the SEM. The Journal of Strength \& Conditioning Research, 19(1): 231-240.

Yüksel, O., M.S. Erzeybek, F. Kaya and M. Gülaç, 2017. Effects of different strength training on body composition in female athletes. Turkey Clinics Journal Sports Science, 9(3): 101-107.

Yüksel, O., B. Gündüz and M. Kayhan, 2019. Effect of crossfit training on jump and strength. Journal of Education and Training Studies, 7(1): 121-124.Available at: https://doi.org/10.11114/jets.v7i1.3896. 